\title{
Direct observation of large electronic domains with memory effect in doped manganites
}

D. D. Sarma*, Dinesh Topwal and U. Manju.

Solid State and Structural Chemistry Unit and Indian Institute of Science, Bangalore - 560012, India.

\author{
S.R. Krishnakumar \\ International Centre for Theoretical Physics (ICTP), \\ Strada Costiera 11, 34100 Trieste, Italy \\ M. Bertolo, S. La Rosa and G. Cautero. \\ Sincrotrone Trieste, S.S. $14 \mathrm{~km} 163.5$ - in AREA \\ Science Park, 34012 Basovizza - Trieste, Italy \\ T. Y. Koo, P. A. Sharma and S-W. Cheong \\ Department of Physics and Astronomy, \\ Rutgers University, New Jersey 08854,USA
}

A. Fujimori

Department of Complexity Science and Engineering, University of Tokyo, 5-1-5 Kashiwanoha, Kashiwa, Chiba 277-8561, Japan 


\begin{abstract}
We use a spatially resolved, direct spectroscopic probe for electronic structure with an additional unique sensitivity to chemical compositions to investigate high quality single crystal samples of $\mathrm{La}_{1 / 4} \mathrm{Pr}_{3 / 8} \mathrm{Ca}_{3 / 8} \mathrm{MnO}_{3}$, establishing the formation of distinct insulating domains embedded in the metallic host at low temperatures. These domains are found to be at least an order of magnitude larger in size compared to previous estimates and exhibits memory effects on temperature cycling in absence of any perceptible chemical inhomogeneity, suggesting long-range strains as probable origin.
\end{abstract}

PACS Nos. 75.47.Lx, 79.60.-i, 71.30.+h, 68.37.Yz 
Doped manganese oxides that exhibit colossal magnetoresistance (CMR) are often characterized by a competition of different electronic phases that critically influence their properties [1-3] and leads to the coexistence of spatially separated competing phases. Despite extensive experimentation [4-8], characteristic length-scales associated with phase coexistence remains an important open question. While theoretical work [9] has pointed to a nanometric length-scale, the first experimental evidence [4] surprisingly indicated a much larger characteristic length, typically $\sim 0.5 \mu \mathrm{m}$. Subsequent experiments [5-8] have uncovered multiple length-scales ranging from the atomic to the sub-micron, covering many orders of magnitude. Additionally, the role of chemical inhomogeneity in driving this phenomenon is not well understood. In this context, it is important to note that these early experiments were carried out on polycrystalline [4] and thin film [6, 7] specimens. The $\mathrm{La}_{1-x-y} \operatorname{Pr}_{y} \mathrm{Ca}_{x} \mathrm{MnO}_{3}$ system is believed to show the clearest example of such phase separation, typically between a ferromagnetic (presumably metallic) and an antiferromagnetic/charge-ordered insulating phase. The recent suggestion [5] of the possibility of an additional ferromagnetic insulating phase opens up further possibilities. In view of this, we investigate the electronic structure of high-quality single crystals of $\mathrm{La}_{1-x-y} \mathrm{Pr}_{y} \mathrm{Ca}_{x} \mathrm{MnO}_{3}$ with $x=y=3 / 8$ using photoemission spectroscopy (PES) with high spatial and energy resolutions. So far the electronic structures of phase-separated manganites have been probed only for $\mathrm{La}_{1-x} \mathrm{Ca}_{x} \mathrm{MnO}_{3}[7]$ and $\mathrm{Bi}_{1-x} \mathrm{Ca}_{x} \mathrm{MnO}_{3}$ [8] using scanning tunneling spectroscopy, which cannot provide any information concerning the chemical composition responsible for the specific electronic structure; therefore, this technique cannot probe the role of chemical inhomogeneity as the primary driving force for the occurrence of phase separation. PES is well known to be the most powerful tool to provide direct information about the electronic structure of almost all varieties of interesting systems. The additional advantage of PES is its unique elemental sensitivity, allowing an accurate determination of chemical composition. Therefore, spatially resolved PES is ideal and unique in its ability to simultaneously probe the chemical homogeneity and spatial variations in electronic structure of such manganites. Interestingly however, this probe has so far not been utilized to investigate the issue of phase separation.

The main impediment in using PES at a sub-micron length scale has been to simultaneously satisfy the demands of a high-energy resolution required to probe the electronic structure relevant to CMR, the ability to deliver a sufficiently intense photon source focused on the sample, and varying the temperature over a wide enough range. These requirements 
becomes even more stringent in view of the need to probe a wide kinetic energy region to cover both valence levels for the electronic structure information and core levels to obtain chemical information at the same time. Recent developments in the synchrotron and associated instrumentation at a third generation source, Elettra at Trieste, Italy, has now made it possible to study the electronic structure by carrying out high resolution $(<50 \mathrm{meV})$ photoemission spectroscopy with a spatial resolution of the order of $0.5 \mu \mathrm{m}$, from temperatures of $40 \mathrm{~K}$ up to room temperature with a photon energy of $95 \mathrm{eV}$ allowing access to a large number of core levels along with the valence level. A schematic of the complete set-up is shown in Fig. 1. A Schwarzschild objective was used to focus the photon flux to a point source on the sample [10]. A large number of independent experiments were carried out to confirm the reproducibility of the results. The temperatures at both the extremes, near $40-50 \mathrm{~K}$ and at the room temperature, could be kept stable for a long time. Images were then acquired with $100 \mathrm{X} 100$ pixels over $\sim 65 \mathrm{X} 32 \mu \mathrm{m}^{2}$ areas at these end temperatures. However, at intermediate temperatures, we recorded coarser images (50 X 50 pixels over the same area), in order to minimize the time to record one image, avoiding possibly larger temperature drifts during a single measurement. The vacuum in the chamber during the measurement and cleaving of the samples was typically $10^{-10}$ mbar.

We have taken a large number of high-resolution spectra over a wide energy range from various points on the sample surface. We have also generated intensity images by recording the spectral intensities over the valence band region with a sixteen-channel detector array. Since these samples do not cleave well, it is not possible to investigate the entire surface at the same level of focus with the electron detection system. Consequently, the absolute intensity at a given energy from any spot on the sample depends on the local topography, particularly the slope and the height, of the specific spot. We use the ratio of the intensity close to the Fermi level to that at the most intense valence band feature at about $3 \mathrm{eV}$ below $\mathrm{E}_{F}$ to partially cancel such topographic contributions. Since the intensity at $\mathrm{E}_{F}$ is expected to be the greatest in the highly metallic state and absent or nearly absent for the insulating state, the mapping of the ratio of intensities at these two energies not only removes the topographic features to some extent, but also accentuates the distinction between the metallic and insulating phases. Essentially, these "ratio" images then identify metallic regions with a large ratio and insulating regions with a small ratio. A comparison of a topographic image, constructed by the total intensity over all the 16 channels, and a 
ratio image clearly establishes which of the features arises from topography and which from a true variation in the electronic structure.

The overall spectral shape of the valence band region, displayed in the inset to Fig. 2a, is typical of the manganites and does not change substantially over most of the energy range with temperature. However, the metal-insulator transition as a function of temperature in the system, consistent with the transport data, is clearly demonstrated in the main frame of Fig. 2(a) with the emergence of a finite spectral intensity at $\mathrm{E}_{F}$ in the low temperature metallic state. In contrast, the high temperature insulating state clearly does not have any intensity at $\mathrm{E}_{F}$ other than contributions arising from various broadening effects, such as thermal and resolution broadening.

In order to understand whether any significant level of chemical inhomogeneity is responsible for electronic phase domain formation in such samples, we have taken a large number of highly resolved spectra over a wide range of energy covering the main valence band consisting primarily of Mn $3 d$ and O $2 p$ states as well as the La $5 p$, Pr $5 p$ and Ca $3 p$ core levels. These spectra were collected with a spatial resolution of $0.5 \mu \mathrm{m}$ from various points on a freshly cleaved crystal surface, and normalized at the highest intensity valence band feature to account for topographic differences. Fig. 2b and 2c show the collection of the valence band and core level spectra, respectively, with 28 spectra in all plotted in each of the panels. It is clear that the spectral features from all these 28 distinct spots on the crystal face overlap each other exceedingly well eliminating the possibility of chemical inhomogeneity at a length scale of $0.5 \mu \mathrm{m}$ or larger in these samples.

Now we present typical electronic structure images from freshly cleaved crystal faces in Fig. 3. The top two rows show images from a particular part, about $54 \mathrm{X} 25 \mu \mathrm{m}^{2}$ area, of the crystal face, with the top-left corner gray-scale image panel showing the topographic image. The remaining panels show the ratio images on a blue (highest)-red (lowest) scale for the ratio of the intensity at $\mathrm{E}_{F}$ to that at the valence band maximum over a thermal cycling as indicated in the figure. This particular area of interest on the sample remained stable throughout our measurements, which was easily confirmed by inspecting the position of topographic features at each temperature. The first panel of the ratio image from the sample at $52 \mathrm{~K}$ shows a predominantly metallic nature judging from the colors ranging from green to deep blue. However, there is clearly an insulating piece signified by the red-yellow region in the middle of the image, which we have surrounded by the thin black 
line for easy identification. Comparing with the topographic image, it is clear that this feature does not arise from any topographic peculiarity, but rather is a true reflection of a distinct electronic structure. The spectrum within this red-yellow region is clearly that of the insulating phase with low intensity at $\mathrm{E}_{F}$ as illustrated in Fig. 2a. This is a clear and direct evidence of the existence of a rather large insulating patch deep within the metallic phase, thereby establishing distinct electronic domains in this material at a low temperature. It is interesting to note that the color contrast vanishes when the sample is warmed up to $253 \mathrm{~K}$ (see figure), thereby showing that the entire sample transforms into the insulating phase at an elevated temperature. Most interestingly, when the sample is again cooled down to the lowest temperature $(50 \mathrm{~K})$, the color contrast reappears, showing metallic regions over most of the area, with the insulating domain at about the same place as before, with about the same orientation but somewhat different morphology. This observation is very significant since it indicates that even at a high temperature, the sample retains an approximate "memory" of the relative locations of the distinct phase domains. This memory effect can only happen if there is some pinning centers that determine the nucleation sites of the insulating domains. We have shown that chemical inhomogeneity is negligible on the length scale of the domains. In addition, a large length scale chemical inhomogeneity would result in a more highly reproducible morphology in contrast to our observation of only an "approximate memory" effect. The fact that the location and orientation are remarkably similar gives credence to the suggestion that primarily long-range strain stabilizes the morphology of these insulating domains within the metallic regime [11].

As shown in the ratio images from 50 through $90 \mathrm{~K}$, it is clear that the contrast in the electronic structure, evident at the lowest temperature, gradually vanishes due to the macroscopic metal-insulator transition with increasing temperature, closely following the metal-insulator transition observed in transport measurements. In order to confirm that these observations are not unique to the particular spot on the sample, we took similar images at the lowest temperature at various places on the crystal surface and in every case we found evidence of such insulating domains deep inside the metallic regime. We illustrate this with another position on the sample surface in the lowest image panels in Fig. 3, recorded as the sample was cycled from the lowest temperature up to about $132 \mathrm{~K}$ and then down to the lowest temperature once again. In the first panel, we show the topographic image; the second panel, showing the corresponding ratio images at a low temperature, exhibits 
an abundance of insulating patches, represented by red-yellow regions marked by thin solid lines, in a predominantly metallic phase represented by blue-green regions. When the sample is heated up to about $132 \mathrm{~K}$, the electronic phase contrasts vanish, as can be seen in the next panel. However, the insulating patches reappear at approximately the same locations when the sample is cycled back to the lowest temperature, supporting the observations stated previously.

It is significant that these observed insulating patches in the metallic regime are large, on the order of several microns across. Such a nearly macroscopic domain formation may appear even more intriguing than the smaller length scale electronic domains (sub-micron or even nano-scale), discussed so far in the literature. The considerably larger size found is presumably due to the high quality of the single crystals used in this work, suggesting that such domains can have even larger sizes for perfect crystals. The present results, establishing a remarkable memory effect in the morphology of the insulating patches, suggest that these insulating domains at low temperatures are initiated by some pinning centers that provide nucleation sites for the insulating phase within the predominantly metallic region. These pinning centers appear to be correlated with long-range strains in the specimen. It is reasonable to expect a larger density of such strain centers (e.g. with a larger defect density) in a crystal of poorer quality or in a polycrystalline material, which then provides a larger density of nucleation sites; this then limits the size of individual patches in such samples. Thus, the wide, orders of magnitude, variation in the length-scale of the phase coexistence phenomena observed in different samples of manganites is most likely due to this variation in the strain center density in different specimens. This further suggests that the intrinsic length-scale may reach as high as several microns, as observed in the present investigation.

We thank ICTP-ELETTRA Users Programme and Department of Science and Technology for support of this investigation. We are grateful to F. Barbo, G. Sandrin and D. Corso for competent technical support. The work at Rutgers was supported by the National Science Foundation, and the work at Tokyo was supported by a Grant-in-Aid for Scientific Research. Floating zone furnace used for the crystal growth was supported by the NSF-MRSEC. U. Manju is thankful to CSIR for support. 
[*] Also at Jawaharlal Nehru Centre for Advanced Scientific Research, Bangalore 560064, India. Electronic address: sarma@sscu.iisc.ernet.in

[1] A. J Millis, P. D. Littlewood, B. I. Shraiman, Phys. Rev. Lett. 74, 5144 (1995); A. J. Millis, B. I. Shraiman, R. Mueller, Phys. Rev. Lett. 77, 175 (1996)

[2] Y. Tokura, Ed., Colossal Magnetoresistance Oxides, Monograph in Condensed Matter Science, Vol. 2, (Gordon and Breach, London, 1999).

[3] D. Akahoshi et al., Phys. Rev. Lett. 90, 177203 ( 2003).

[4] M. Uehara, S. Mori, C. H. Chen, S.-W. Cheong, Nature 399, 560 (1999).

[5] J. C. Loudon, N. D. Mathur, P. A. Midgley, Nature 420, 797 (2002)

[6] L. Zhang, C. Israel, A. Biswas, R. L. Greene, A. de Lozanne, Science 298, 805 (2002).

[7] M. Fath et al., Science 285, 1540 (1999).

[8] Ch. Renner, G. Aeppli, B.-G. Kim, Y.-Ah. Soh, S.-W. Cheong, Nature 416, 518 (2002).

[9] A. Moreo, S. Yunoki, E. Dagotto, Science 283, 2034 (1999).

[10] F. Barbo et al., Rev. Sci. Instrum. 71, 5 (2000).

[11] K. H. Ahn, T. Lookman, A. R. Bishop, Nature 428, 401 (2004) 


\section{Figure Captions}

Fig. 1 : The schematic of the experimental setup. A Schwarzschild objective focuses the high brilliance synchrotron beam to a sub-micron spot onto the sample and the photoemitted electrons are energy analysed with a hemispherical electron energy analyzer and a position sensitive detector. The sample stage is manipulated with piezo drives to scan the sample surface. A continuous helium flow cryostat allows the sample temperature to be varied between approximately 40 and $300 \mathrm{~K}$. The total intensity from each spot can be mapped to provide a microscopic view of the sample surface that includes contributions from the topography as well as variations in the electronic structure. Taking the ratio of the intensities at two different energies and plotting the ratio instead of the absolute intensity in order to accentuate only the changes in the electronic structure can largely eliminate topographic features.

Fig. 2 : (a) Spectral changes close to the Fermi energy across the metal-insulator transition, exhibiting a finite density of states at $\mathrm{E}_{F}$ in the metallic phase. The inset shows the overall spectral feature over a wide energy range. (b) Superposition of valence band spectra consisting of mainly Mn $3 d$ and O $2 p$ states recorded with a high spatial resolution $(0.5 \mu \mathrm{m})$ at 28 different spots on the sample. (c) Superposition of the shallow core level spectra from La, Pr and Ca from the same set of spots as in panel (b).

Fig. 3 : Spectromicroscopic images over $\sim 54 \mathrm{X} 25 \mu \mathrm{m}^{2}$ areas of the sample surface. The top two rows show images from one region of the sample and the bottom row from another part, with the first panel in each of the two sets represented by gray scale being a topographic image and the rest ratio images. Ratio images at the lowest temperature for each image show emergence of distinct insulating patches (red-yellow) deep within the metallic regime (blue-green), which exhibit memory effects in the formation and morphology on temperature cycling. The color contrast in the ratio images vanishes and the entire sample transforms into the insulating phase as the temperature is increased, converting the system into an electronically homogeneous phase. 


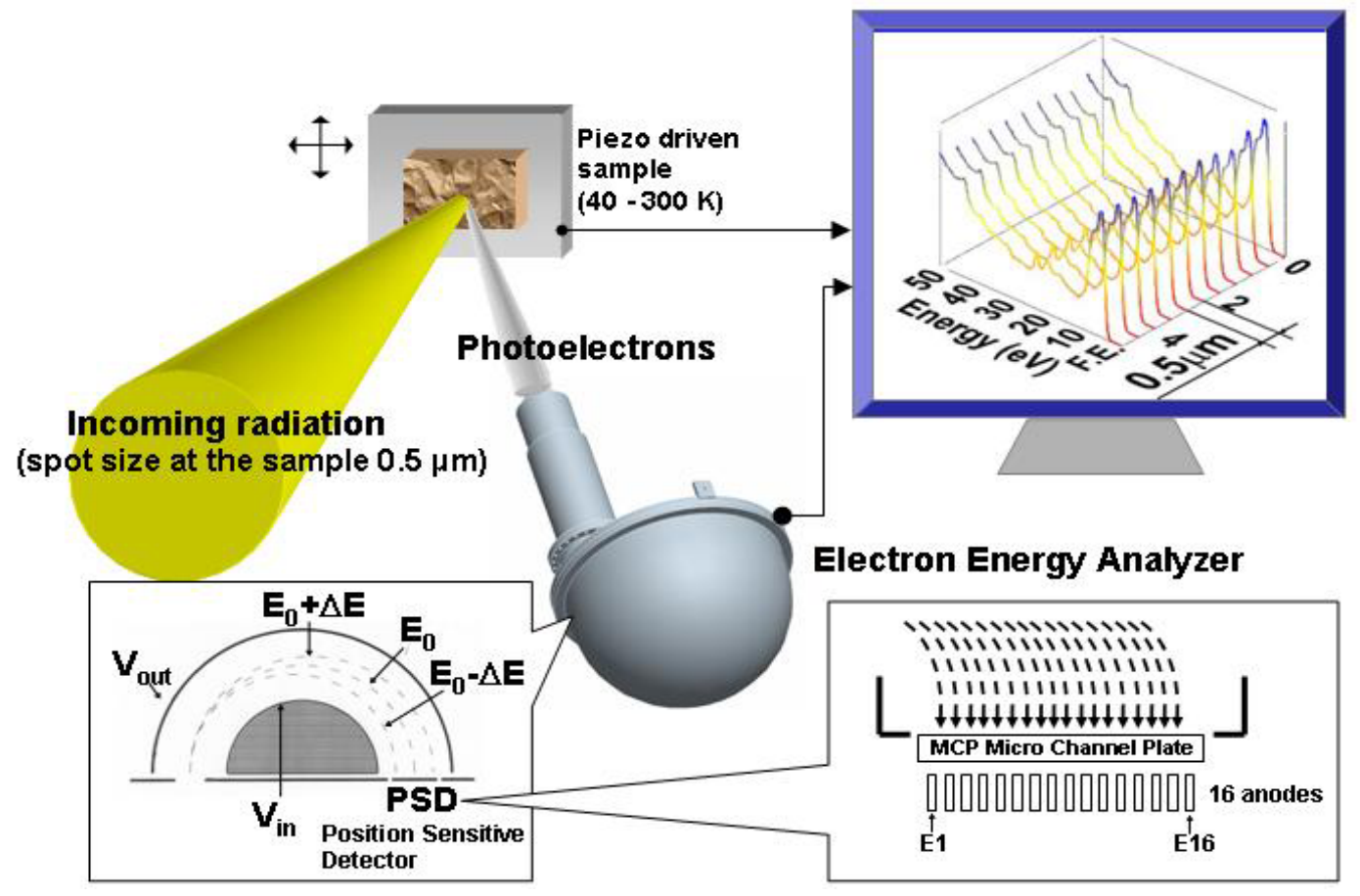

Sarma et al. Fig 1. 


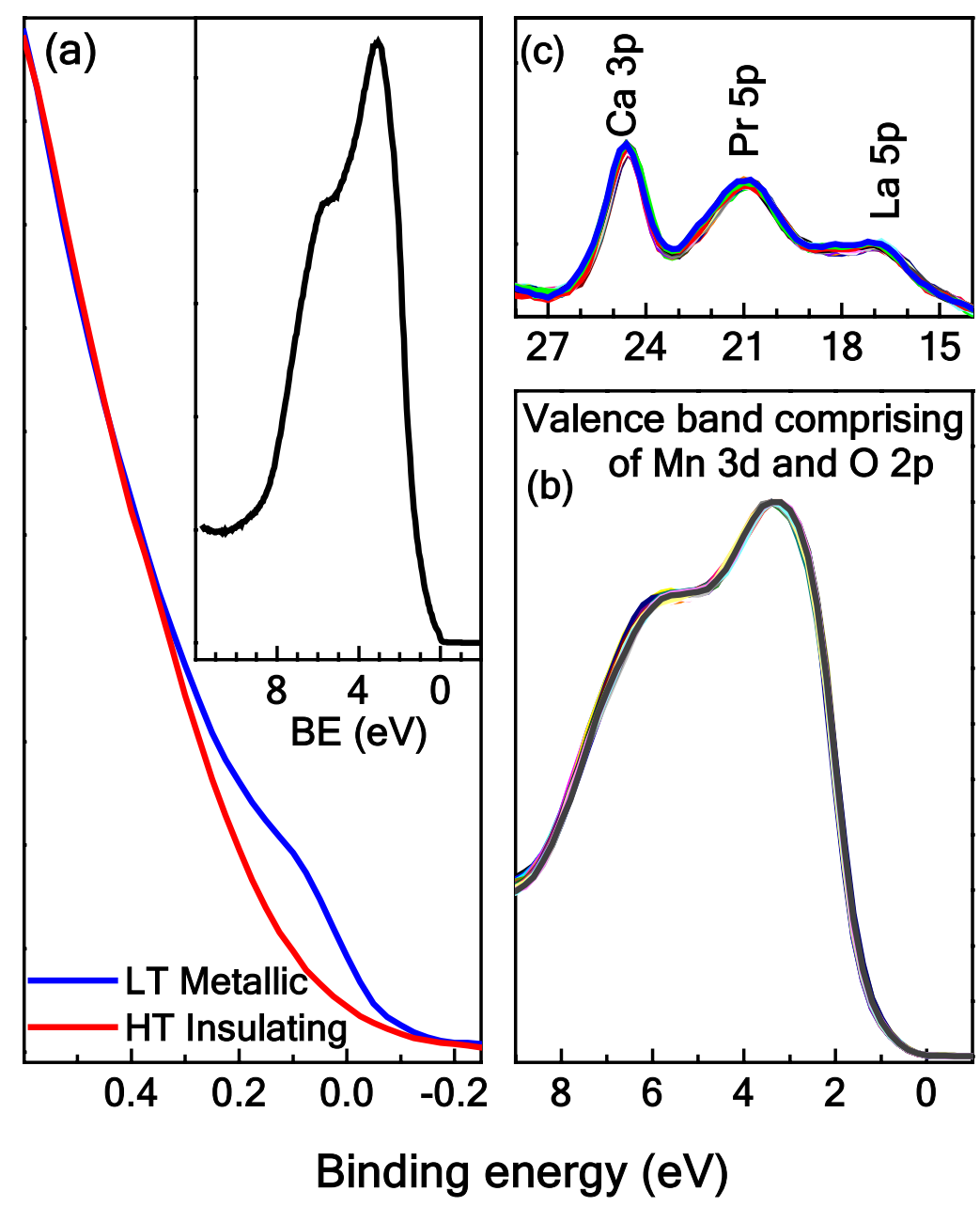

Sarma et al. Fig 2. 


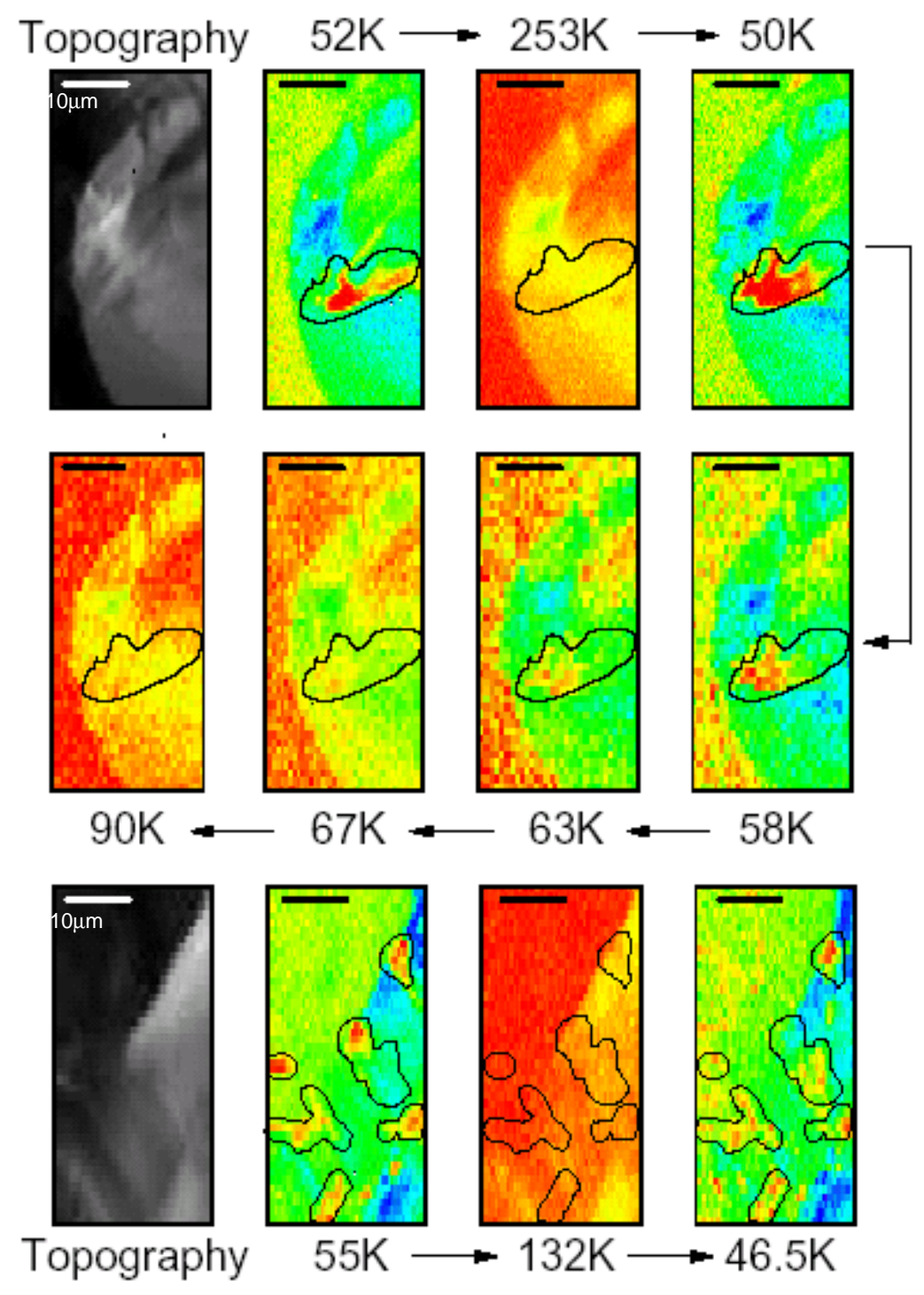

Sarma et al. Fig 3. 